Aus dem Medizinhistorischen Institut der Universität Zürich

(Direktor: Prof. Dr. med. Erwin A. Ackerknecht)

\title{
Ein Hinweis auf die Bergkrankheit in Europa aus dem 14. Jahrhundert*
}

\section{Von Eduard-Rudolf Müllener, Zürich}

In der ersten der sogenannten Berg- oder Höhenkrankheit gewidmeten Monographie aus dem Jahre 1854 beschrieb der Zürcher Arzt Conrad Meyer-Ahrens (1813-1872) ihre Symptome, die bei Aufenthalten in Höhen über etwa $3500 \mathrm{~m}$ ü.M. auftreten können, mit folgenden Worten: «Die wesentlichsten, d.h. die am gewöhnlichsten vorkommenden Erscheinungen der Bergkrankheit, sind bei den Menschen: Ekel, Abneigung gegen Speisen, meist auch gegen den Wein ... starker Durst ... Übelkeiten mit Erbrechen. Beschleunigtes, keuchendes Athmen, beschleunigter Kreislauf, Pulsiren großer Arterien, z. B. der Schläfenarterien, Herzklopfen, heftige Oppression, Erstickungsangst, Schwindel, heftige Kopfschmerzen, Anwandlungen von Ohnmacht, unbezwingbare Schläfrigkeit ... Endlich außerordentliche, wahrhaft fabelhafte Erlahmung der Muskeln.» ${ }^{1}$ Zur Ätiologie bemerkte er: «... nach meiner Ansicht ... (spielen) die Hauptrolle die absolute Abnahme des Sauerstoffgehaltes in der dünnern Luft, die raschere und quantitativ bedeutendere Wasserverdampfung und die intensive Einwirkung des vom Schnee zurückgeworfenen und direct auffallenden Lichts, während ich dagegen in ätiologischer Beziehung der directen Einwirkung des verminderten Luftdruckes nur einen untergeordneten Rang einräumen möchte ... Viele Symptome, die Beschleunigung des Pulses, der Schwindel, die Anwandlungen von Ohnmacht, die Neigung zum Schlafe, die Erlahmung der Muskeln usw. erinnern lebhaft an den Symptomenkomplex der Anämie. » ${ }^{2}$ Meyers Vermutung, der Krankheit liege - nebst andern Faktoren, die später als unwesentlich erkannt wurden - ein Sauerstoffmangel zugrunde, eine Ansicht, die erstmals Alexánder von Humboldt 1802 auf Grund von Sauerstoffmessungen in großer Höhe in wissenschaftlich fundierter Form

\footnotetext{
*Herrn Prof. Dr.med. Dr.phil. Dr.med.h. c. Alexander von Muralt zum 60. Geburtstag gewidmet.

${ }^{1}$ C. Meyer-Ahrens, Die Bergkrankheit oder der Einfluß des Ersteigens großer Höhen auf den thierischen Organismus, Leipzig 1854, S. $100 . \quad{ }^{2}$ Ibid., S. $132 \mathrm{f}$.
} 
äußerte ${ }^{3}$, wurde vom Franzosen Denis Jourdanet in den siebziger Jahren des letzten Jahrhunderts tierexperimentell überprüft, wobei aber Jourdanet seine eigenen Untersuchungen noch nicht völlig beweisend erschienen ${ }^{4}$. In dem klassischen Werk La pression barométrique gelangte dann sein Landsmann Paul Bert zum zwingenden Schluß, daß der in größerer Höhe erniedrigte Luftdruck ausschließlich über eine verminderte Sauerstoffspannung der Atemluft die Symptome der Berg- oder Höhenkrankheit hervorruft $^{5}$. Sowohl Meyer wie Bert behandeln historische Aspekte der Höhenkrankheit und sind sich darin einig, daß der Jesuitenpater José DE Acost A im ausgehenden 16. Jahrhundert als erster die entsprechenden Erscheinungen beschrieb, die ihn und seine Gefährten im Jahre 1581 während einer Reise in Peru überfielen; Acosta spricht von einem «tödlichen und sonderbarèn Übel» mit Schmerzen und Erbrechen, welches bei der Besteigung eines Berges namens Periacaca auftrat, 3 bis 4 Stunden lang anhielt und nach dem Abstieg rasch wieder verschwand. Er schreibt die Krankheit der dünnen Luft sowie der Kälte zu, welche die Lebenskraft untergrüben ${ }^{6}$. Die Theorie von der zu dünnen Luft, die ihren Druck verliert, was wiederum zu einer Ausdehnung des Bluts in den Blutgefäßen sowie gelegentlich zu Gefäßrupturen (Hämoptoe) führen sollte, wurde im 17.Jahrhundert von ROBERT BOYLE mittels tierexperimenteller Untersuchungen erhärtet und behielt ihre Geltung bis ins 19. Jahrhundert?

${ }^{3}$ Nach M. Mumenthaler, Die Erscheinungen des Sauerstoffmangels, ein geschichtlicher Beitrag, Gesnerus 16 (1959) 47-65, $59 . \quad{ }^{4}$ D. Joundanet, Influence de la pression de l'air sur la vie de l'homme, 2 Bände, Paris 1875, Band 1, S. 181.

5 P. Bert, La pression barométrique, Paris 1878, S. 1081 : «Nous avons exposé... les preuves de cette vérité que la diminution dans la pression barométrique n'agit sur les êtres vivants qu'en diminuant la tension de l'oxygène qu'ils respirent...»

${ }^{6}$ José de Acosta, Historia Natural y Moral de las Indias: en que se trata de cosas notables del Cielo, de los elementos, metales, plantas y animales etc., Sevilla 1590, zit. nach BERT (siehe Anmerkung) S. 25-7, und MeYer (siehe Anmerkung 1) S. 11.

7 R. BoyLe, New experiments physico-mechanicall, touching the spring of the air, and its effects, made, for the most part, in a new pneumatical engine, Oxford 1660 , zit. nach L.L. LANGLEY, An historical introduction to the physiology of anoxia, Bull. Hist. Med. 14 (1943) 321-40, 324f. Die Theorie von der zu dünnen Luft und der Ausdehnung des Blutes findet sich u. a. auch bei ЈонаNN ЈАков ScheUchzer, der allerdings aus religiösen Gründen nicht an die Existenz einer eigentlichen Bergkrankheit glauben wollte: «Ich kann mir aber nicht einbilden ... daß die zu des Menschen Nuz geordnete Gebirge solten von dem Schöpfer so hoch aufgeführet worden sein / daß die darauf steigende Menschen oder Thiere solten ... eine Ruptur oder Zerspringung ihrer Aderen austehen / und damit in Lebensgefahr lauffen» (J.J. Scheuchzer, Natur-Historie des Schweizerlandes, 3 Bände, 2.Auflage, Zürich 1752, Band 1, S. 12). Sein Bruder JohanN Scheuchzer scheint im 
Die Frage, ob die Bergkrankheit oder einzelne ihrer Symptome nicht schon vor Acosta bekannt waren, erscheint berechtigt, insbesondere wenn man an die in der Renaissance erfolgte «Entdeckung der Landschaft» denkt. In Jacoв Burckhardts Die Kultur der Renaissance in Italien findet sich denn auch ein entsprechender kurzer Hinweis auf einen europäischen Beitrag aus dem 14. Jahrhundert, der einer etwas ausführlicheren Besprechung würdig erscheint ${ }^{8}$. Burckhardt erwähnt das Werk Dittamondo des Fazio degli Uberti, eine gereimte Kosmographie aus der Mitte des 14. Jahrhunderts, worin bei der Schilderung einer Bergbesteigung bereits Phänomene beschrieben werden, die sich erst etwa ab $3000 \mathrm{~m}$ ü.M. einstellen. Fazio (Bonifazio) degli Uberti, ein wahrscheinlich um etwa 1400 in Pisa geborener Dichter, aus dessen Leben wenig mehr bekannt ist, als daß er im Dienste der Visconti, der Scaglieri und eventuell der Carraresi stand (verstorben kurz nach 1369 in Verona), gibt in seinem 1330 bis 1360 entstandenen, später umgearbeiteten, aber nie vollendeten Hauptwerk Dittam ndo (dicta mundi), einer fingierten visionären Reisebeschreibung im Stile DA $\$ TES, eine Übersicht über das Wissen seiner Zeit vom Wesen, der Schönheit und der Geschichte der Welt ${ }^{9}$. In diesem Sinne durchwandert er in dem in Terzinen geschriebenen Gedicht, begleitet von seinem mythischen Führer Solxv us ${ }^{10}$

Jahre 1709 bei einer Bergbesteigung in Graubündea einige Anzeichen der Höhenkrankheit wahrgenommen zu haben und spricht in seinem Bericht davon, daß er die Vorboten der unmittelbar bevorstehenden Hämoptyse verspärte - was zeigt, daß er zweifellos an die Theorie glaubte (Disquisitionis physicae de mete , ris aqueis pars prina, sistens muteriam de vaporibus, nebulis et nubibus / pro examine philos $v$ piico rite consequendo sub praesidio Johannis Scheuchzeri, M.Doct., Professoris Philosop iie Naturalis Ordinarii, Tiguri 1736, S. 39f.). Es ist dies wohl der erste Hinweis auf das mögliche Vorkommen der Höhenkrankheit in der Schweiz. Auch HaLler vertritt die oben erwähnte Theorie, glaubt aber, daß die Berge in der Schweiz nicht hoch genug seien (er erwähnt u.a. den Iurkapaß), um entsprechende Symptome hervorzurufen (A. HALLER, Elementa Physiologiae corporis humani, Band III, Lausanne 1764, S. 196 f.).

8 J. BurckhardT, Die Kultur der Renaissance in It alien, Band 2, 10. Auflage, 1908, S. 20. Die erste Auflage erschien 1860, und es ist nicht unwahrscheinlich, daß die 1854 publizierte Monographie von Meyer-Ahrens die Aufmerksamkeit BurckHardTs auf die entsprechenden Erscheinungen lenkte.

${ }^{9}$ Nach Enciclopedia italiana di scienze, lettere ed arti, Band 34, Rom 1936-1945, S. 584. Als Quellen dienten Uberti u. a. Solinus, Plinius der Ältere, Isidor von Sevilla und Pomponio Mela.

${ }^{10}$ C. Iulius Solinus. Genaue Lebensdaten unbekannt, lebte wahrscheinlich in der Mitte des 3. Jh.s n. Chr. in Rom. Seine Erdbeschreibung Collectanea rerum memorabilium stützt sich auf Plinius den Ältenen und Pomponio Melas Corografia (nach Enciclopedia italiana etc., Band 32, siehe Anmerkung 9, S. 77 f.). 
(der bei ihm die Stelle von Dantes Vergil einnimmt) Italien, Griechenland, Germanien, Frankreich, Spanien, Nordeuropa, Afrika und Vorderasien. Bei der Besteigung des Olymp schildert er folgende Begebenheit:

Io non fui su per quelle vie rimote, ch'ogni mio poro si converse in fonte, e acqua venni dal capo a le piote.

Ma poi ch'io fui al sommo del gran monte, dove posar credea e prender lena, io mi sentio gravar gli occhi e la fronte, e'l sangue spaventar per ogni vena, tremare il cuore, e venni freddo e smorto come chi giunge all'ultima sua pena.

Solino allora, sí come uomo accorto, misemi al naso una bagnata spunga per la cual presi subito conforto:

«Piú non temer che l'accidente giunga, però che qui trovâr questo argomento quei buon che veder volsono a la lunga.» Come fuor mi sentivo d'ogni spavento, con le mie guide e con la spunga al naso mi mossi tutto ancor debole e lento ${ }^{11}$.

In freier Übersetzung:

Kaum befand ich mich auf diesen abgelegenen Wegen, da verwandelte sich jede meiner Poren in einen Brunnen, und Wasser kam vom Kopf bis zu den Fußsohlen.

Als ich mich aber dann auf dem Gipfel des großen Berges befand, wo ich hoffte, ausruhen und Atem holen zu können, fühlte ich meine Augen und die Stirne schwer werden Und das Blut in erschreckender Weise durch jede Ader jagen, das Herz zittern, und ich wurde kalt und aschfahl wie einer, der der letzten Strafe entgegengeht.

Solinus aber, wie es sich für einen scharfsinnigen Mann gehört, hielt mir einen benetzten Schwamm vor die Nase, wodurch ich rasch erleichtert wurde.

11 Fazio degli Uberti, Il Dittamondo e le Rime. A cura di Giuseppe Corsi, Band 1, Bari 1953, S. 266 (Dittamondo Libro IV, Cap. 4, Zeilen 76-93). 
«Nun ist nichts mehr zu fürchten, denn die hierin enthaltene Kraft wird alles mit der Zeit zum Guten wenden.»

Da ich mich von jedem Schreck befreit fühlte, mit meinen Führern und dem Schwamm vor der Nase, bewegte ich mich einstweilen noch ganz schwach und langsam weiter.

Schon Aristoteles lehrte angeblich, auf dem Gipfel des Olymp sei die Luft so dünn, daß ein Mensch dort nur mit Hilfe eines vor die Nase gehaltenen durchnäßten Schwamms überleben könne ${ }^{12}$. Die «Therapie» hat Fazio degli Uberti also wahrscheinlich der antiken Überlieferung entnommen. Dieselbe Überlieferung mag ihn auch dazu bewogen haben, das Auftreten der Bergkrankheit gerade bei der fingierten Besteigung des Olymp zu schildern. Seine Beschreibung geht jedoch weit über die kurze Bemerkung von Aristoteles hinaus und hebt einige typische Symptome so klar hervor (allgemeine Ermattung, Kopfschmerzen in Stirn- und Augengegend, Herzklopfen und jagende Pulse, Kältegefühl und Hautverfärbung [Zyanose ?]), daß sie jener von Acosta kaum nachsteht und die Annahme nahelegt, die Bergkrankheit sei schon im Europa des 14. Jahrhunderts bekannt gewesen. Fazio degli Uberti wird ihre Phänomene kaum am eigenen Leibe erlebt haben - aber offensichtlich hat er darüber recht genaue Berichte vernommen. Er scheint auch gewußt zu haben, daß die Symptome nur in recht großer Höhe auftreten, bemüht er sich doch, dem Leser den Olymp als wirklich ausgesprochen hohen Berg darzustellen:

Forse otto miglia era ito, ch'io compresi

un monte innanzi a me, ch'era alto tanto, che indarno l'occhio a la cima sospesi.

Allor mi volsi dal mio destro canto

e dimandai Solin: «Che monte è questo,

che sopra ogni altro si puote dar vanto ? ${ }^{13}$

12 Nach R. Boyle, Nova experimenta Physico-Mechanica de vi aeris elastica et ejusdem effectibus, facta maximam partem in nova machina pneumatica, Oxoniae 1661, S. 244: "Quod eorum nonnulli qui altitudinem montium tractant, ex Aristotelem commemorant, eos scil: qui ad summitatem Olympi ascendunt, vix vivere posse nisi spongias secum portent humidas, quarum ope Aerem illum insugere possent, alias Respirationi, prae tenuitate, inutilem ...»

${ }^{13}$ Fazio Degli Uberti, l.c., siehe Anmerkung ${ }^{11}$, S. 75 (Dittamondo Libro IV, Cap. 4, Zeilen 58-63). 


\section{In freier Übersetzung:}

Ungefähr acht Meilen waren wir gegangen, als ich vor mir einen Berg gewahrte, der so hoch war, daß ich vergebens mit meinem Auge nach dem Gipfel suchte.

Da wandte ich mich zur Rechten und fragte

Solin: «Welcher Berg ist dies, der sich über alle andern rühmen kann?»

Solinus antwortet dann, es sei der Olymp.

Ferner verwechselt Fazio degli Uberti auch nicht die Symptome der Bergkrankheit mit der bei Bergbesteigungen auftretenden gewöhnlichen Müdigkeit, die ihm ebenfalls bekannt ist und welche er an anderer Stelle bei der Beschreibung der Wanderung auf den Parnaß folgendermaßen schildert:

Non era al mezzo, quando stanco e roggio sí venni, ch'io 'l chiamai piú d'una volta ché innanzi m'era: «Attenti, ch'io m'appogio.»

Wir waren noch nicht auf halber Höhe angelangt, als ich so müde und erschöpft ward, daß ich ihm, der vor mir war, mehrmals zurief: «Halt, ich muß mich setzen!»

Solinus dreht sich dann jeweils um und hilft ihm weiter.

Aus den zitierten Stellen ergibt sich, daß zweifellos einige Symptome der Berg- oder Höhenkrankheit bereits im Europa des 14. Jahrhunderts, also rund zweihundert Jahre vor ihrer Beschreibung durch Acosta, recht gut bekannt waren.

${ }^{14}$ l.c., S. 243 (Dittamondo Libro III, Cap. 21, Zeilen 13-15).

${ }^{15}$ Direkte Hinweise auf die Ersteigung hoher Berge aus jener Zeit sind allerdings äußerst spärlich. Eine entsprechende Notiz (leider ohne Literaturangaben) findet sich bei ZunTz: «Auch war während des Mittelalters der Sinn für die Schönheit des Hochgebirges nicht völlig verlorengegangen ... Selbst von eigentlichen Bergtouren wird uns berichtet. So wurde im Jahr 1358 die Roccia Melone $(3537 \mathrm{~m})$ bei Susa von Rotario d'Asti bestiegen, der dann sogar, um ein Gelübde zu lösen, nahe dem Gipfel eine Kapelle erbaute. Diese Kapelle wurde in der Folge ein oft besuchter Wallfahrtsort. Ferner wird uns von einer rein touristischen Besteigung des Pic du Canigou in den Pyrenäen durch PEter III. von Aragonien (Ende des 13. Jahrhunderts) erzählt.» (N.Zuntz, A. Loewy, F. Müller und W. CASPaRI, Höhenklima und Bergwanderungen in ihrer Wirkung auf den Menschen, Berlin 1906, S. 10.) 